Article

\title{
Sensorless Control of Dual-Active-Bridge Converter with Reduced-Order Proportional-Integral Observer
}

\author{
Duy-Dinh Nguyen ${ }^{1,2, *(\mathbb{D})}$, Dinh-Hoa Nguyen ${ }^{3}{ }^{(\mathbb{D})}$, Toshihisa Funabashi ${ }^{4}$ and Goro Fujita ${ }^{1}$ \\ 1 Department of Electrical Engineering, Shibaura Institute of Technology, Tokyo 135-8548, Japan; \\ gfujita@shibaura-it.ac.jp \\ 2 School of Electrical Engineering, Hanoi University of Science and Technology, Hanoi, Vietnam \\ 3 International Institute for Carbon-Neutral Energy Research (WPI-I2CNER), Kyushu University, \\ Fukuoka 819-0395, Japan; hoa.nd@i2cner.kyushu-u.ac.jp \\ 4 Institute of Materials and Systems for Sustainability (IMaSS), Nagoya University, Nagoya 464-8603, Japan; \\ funabashi@imass.nagoya-u.ac.jp \\ * Correspondence: nguyen.duy.dinh.g7@shibaura-it.ac.jp; Tel.: +81-03-5859-8206
}

Received: 26 March 2018; Accepted: 10 April 2018; Published: 13 April 2018

\begin{abstract}
When controlling a Dual-Active-Bridge (DAB) DC/DC converter, the high frequency terminal current is usually measured for use in the current feedback controller. In order to measure that current, a wide bandwidth sensor accompanied with high-speed amplifiers are required. Furthermore, a high Analog-to-Digital sampling rate is also necessary for sampling and processing the measured data. To avoid those expensive requirements, this paper proposes an alternative control method for the DAB converter. In the proposed method, the terminal current is estimated by a reduced-order proportional integral observer. A technique is also proposed to reduce the phase drift effect when the voltages at two terminals are not matched. Afterwards, a combined current feedforward-voltage feedback control system is developed to enhance the system dynamics and to regulate the output voltage. This control system needs only the information of the terminal voltages and no current sensor is required. Experimental results show that the observer can estimate the terminal current very quickly with the accuracy of more than $98 \%$. In addition, the output voltage is well regulated with a fluctuation of less than $\pm 2.6 \%$ and a settling time of less than $6.5 \mathrm{~ms}$ in the presence of a $30 \%$ load change.
\end{abstract}

Keywords: dual-active-bridge DC/DC converter; reduced-order observer; proportional integral observer; feedforward current control; observer-based control system

\section{Introduction}

In micro-grid applications, line frequency transformers (LFTs), in correspondence with mechanical breakers, are usually employed to interconnect a micro-grid with the main grid. LFTs work at the line frequency to perform $\mathrm{AC}$ to $\mathrm{AC}$ power conversion. However, the deep penetration of renewable energy causes negative effects to the distribution line, e.g., voltage fluctuation, frequency variation, etc. The undesired fluctuations may be transferred to the downstream or upstream utilities via LFTs and cause excessive operation of mechanical voltage stabilizers [1].

In order to improve the power quality, reliability and stability, there are some possible solutions, such as adopting Flexible Alternating Current Transmission System (FACTS) devices [2] to process the fluctuations at the point of common coupling or using Solid-State-Transformers (SSTs) [3] instead of LFTs. Among those, the SST, which is depicted in Figure 1, appears to be a promising device to resolve the aforementioned problems.

An SST consists of three main parts: an active rectifier, a bidirectional Dual-Active-Bridge (DAB) DC/DC converter and an inverter. Compared to LFTs, SSTs do not only allow galvanic isolation and 
bidirectional power transmission but can also be used for all kinds of electric power conversion, not only AC-AC conversion. Furthermore, SSTs can perform asynchronous interconnection, voltage/load regulation, protection, etc. Those features obviously show the advantages of SSTs over LFTs. To achieve all the capabilities of SSTs above, the underlying key component is the Dual-Active-Bridge (DAB) converter whose structure is depicted in Figure 1. A high-frequency isolated transformer is used to separate two DC sides. Each winding of the transformer connects to an inverter. Since the switching frequency is usually from several $\mathrm{kHz}$ to hundreds of $\mathrm{kHz}$, the overall size can be reduced and the power density can be increased [4].

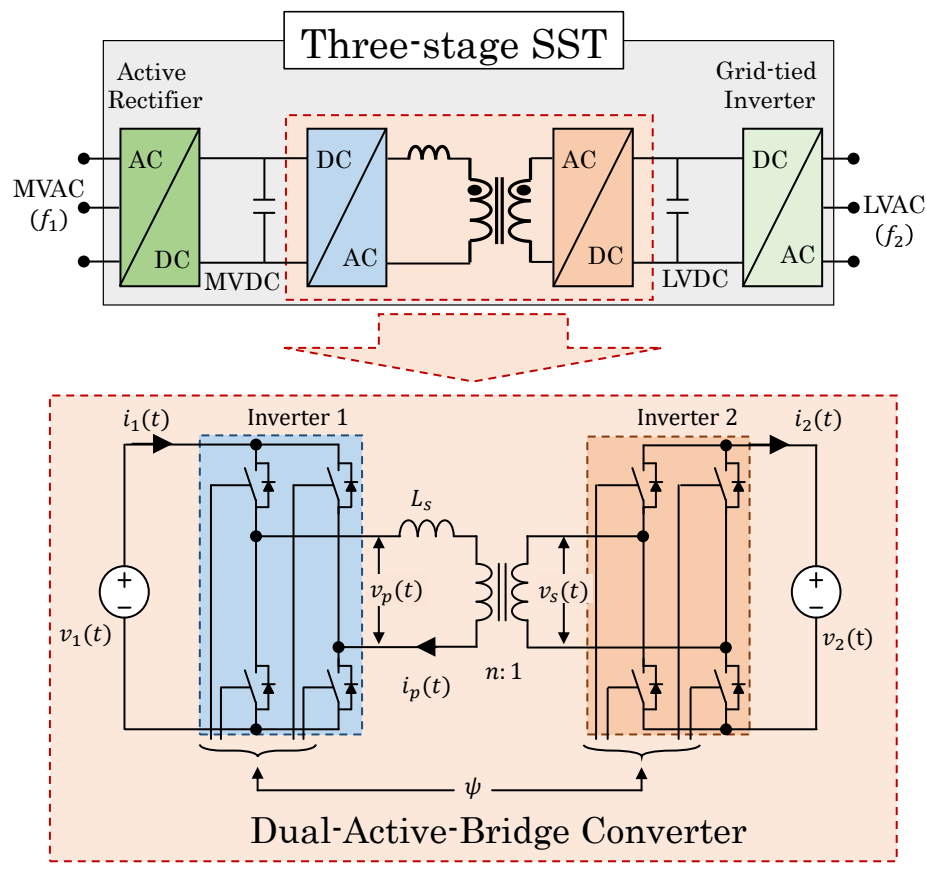

Figure 1. Three-stage Solid State Transformer (SST).

In terms of dynamic control for DAB converters, there are two modes of control that are usually employed: voltage mode and current mode [5-12]. Assume that power transmission is from the medium voltage DC (MVDC) side to the low voltage DC (LVDC) side. When the low voltage AC (LVAC) utility is not available, the SST should generate AC voltage to supply the LVAC bus, then the $\mathrm{DAB}$ converter will act as a voltage source to regulate the LVDC bus. In this voltage mode, the load current is usually used for enhancing the system dynamics. Examples of this control technique are many, such as the study reported in [6] that utilized the load current as a feedforward signal to enhance the system dynamics; the control strategy proposed in [7] did not feedforward the current but used it to derive the optimal phase shift for current-stress minimization, etc. In contrary, in the interconnected mode, the LVDC bus is regulated by the grid-tied inverter; thus, the DAB converter should behave as a current source. In this context, a feedback current mode controller is usually employed. For example, in [8], the LVDC current was necessary for the dual-loop cascade controller; in [9], the instantaneous primary current was sampled at a certain instant for use in a predictive controller, etc.

It is worth noting that the control techniques mentioned in [6-9] required the information of the terminal current or the transmission current for the control system in one way or another. The two currents, one is AC at the switching frequency whereas the other is DC but contains a high ripple at double switching frequency, require expensive and high bandwidth current transducers to be measured. In addition, a high sampling rate Analog-to-Digital conversion is also required to sample the signal. To ensure the accuracy, according to the Nyquist theorem, the sampling rate should be at least four times faster than the switching frequency, which may be impossible in many cases where the switching frequency is in the range of hundreds $\mathrm{kHz}$. Moreover, the current sensors are sensitive 
to noises, and hence extra efforts are needed for calibrating and filtering. For the aforementioned reasons, eliminating current sensors, by means of sensorless current control, can significantly simplify the signal processing procedures and save system resources for other tasks, e.g. modulation, control procedures, communication, etc. The above reasons provide motivation for the study on sensorless control structure in this paper.

In fact, the sensorless current control technique has been popularly applied for unidirectional DC/DC converters [13-15]. Recently, it is also applied for controlling DAB converters [16-19]. In [16], the load current is estimated by a nonlinear disturbance observer (NDO) and is then fed-forward to the voltage control loop to improve the dynamic performance. Although the NDO can estimate the current, the design procedures seem to be relatively complicated with many design parameters. The control system reported in $[17,18]$ received the relevant data from the front-end rectifier, which means that the control bandwidth will be dependent on the communication speed. Furthermore, if there are other devices such as batteries or photo-voltaic panels connected to the MVDC bus, this method will no longer be applicable. In [19], a set of mathematical equations was established to calculate the terminal current. Nevertheless, there was no dynamic model for further control system design since the equations were based on the steady state analysis.

From another aspect, the techniques reported in $[20,21]$ used observers to estimate the active and reactive powers, and then a decoupled current controller was employed to regulate two power components individually. However, the observer proposed in [20] requires the terminal current while that proposed in [21] needs the transmission AC current, as input information. Furthermore, the observer models in $[20,21]$ were established based on some approximations. When the voltage ratio is different from unity, the accuracy of those approximations decreases due to the distortion of the current waveform.

This paper then advances the observer-based control strategy presented in [20] in several aspects:

- First, the current sensors are removed and an observer is developed to estimate the terminal current, which renders our proposed control structure sensorless.

- Second, to reduce the computational burden, a reduced-order proportional integral observer (RPIO) is proposed since terminal voltages can be measured directly with low bandwidth transducers.

- Third, a compensation technique is proposed to minimize the influence of the phase drift effect (due to waveform distortion of the AC current when terminal voltages are not matched) on the observation performance.

- Finally, a combined current feedforward and voltage feedback control system is developed to regulate the secondary terminal voltage.

As shown later, the proposed RPIO can help estimate the load current with the accuracy of higher than $98 \%$ without any current sensors. By feeding-forward the estimated load current to the combined current feedforward-voltage feedback controller, the system dynamics are significantly improved. Voltage fluctuation as well as the setting time are reduced by approximately $50 \%$ in the presence of a $30 \%$ load change compared to that when the feedforward path is not applied. Computation burden of the proposed RPIO is, however, a little bit increased compared to that of the reduced-order observer reported in [20] because of a higher order of the observer model (3 vs. 2). Nevertheless, the expensive current sensor is removed and thus efforts to calibrate that noisy signal are also eliminated and economical benefit is also achieved.

\section{DAB Small Signal Model}

In order to simplify the analysis, the primary referred diagram depicted in Figure 2 is employed. The Fundamental Harmonic Approximation method is utilized to model the system because of its effectiveness and high accuracy [22]. In Figure $2, v_{p e}(t), v_{s e}^{\prime}(t)$ and $i_{p e}(t)$ represent the voltages across 
the primary and secondary windings of the transformer, and the primary current, respectively, which are determined by:

$$
\left\{\begin{array}{l}
v_{p e}(t)=\frac{4}{\pi L_{s}} v_{1}(t) \sin \omega_{s} t \\
v_{s e}^{\prime}(t)=\frac{4 n}{\pi L_{s}} v_{2}(t) \cos \psi \cos \omega_{s} t+\frac{4 n}{\pi L_{s}} v_{2}(t) \sin \psi \sin \omega_{s} t \\
i_{p e}(t)=i_{d}(t) \cos \omega_{s} t+i_{q}(t) \sin \omega_{s} t
\end{array}\right.
$$

where $v_{1}(t)$ and $v_{2}(t)$ are terminal voltages; $L_{s}$ is the leakage inductance of the transformer; $n$ is the winding ratio, in this paper, $n=1 ; \omega_{s}$ is the angular switching frequency; $i_{d}(t)$ and $i_{q}(t)$ are the direct and quadrature components of the transferred current; and $\psi$ is the phase shift.

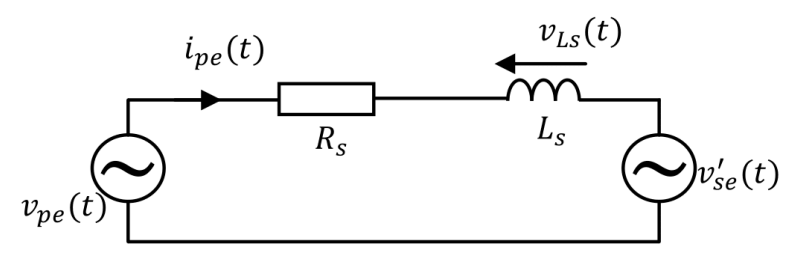

Figure 2. Primary referred diagram.

According to [23], the large signal model of the DAB converter is:

$$
\left\{\begin{array}{l}
\frac{d i_{d}(t)}{d t}=-\omega_{p} i_{d}(t)-\omega_{s} i_{q}(t)+\frac{4 v_{1}(t)}{\pi L_{s}}-\frac{4 n v_{2}(t)}{\pi L_{s}} \cos \psi, \\
\frac{d i_{q}(t)}{d t}=\omega_{s} i_{d}(t)-\omega_{p} i_{q}(t)-\frac{4 n}{\pi L_{s}} v_{2}(t) \sin \psi, \\
\frac{d v_{2}(t)}{d t}=\frac{i_{2}(t)}{C_{o}}-\frac{i_{R}(t)}{C_{o}}
\end{array}\right.
$$

where $\omega_{p}=\frac{R_{s}}{L_{s}} ; R_{s}$ is the resistance referred to the primary side of the transformer; $i_{R}(t)$ is the load current; $C_{o}$ is the output capacitor; and $i_{2}(t)$ is the output DC current of the Inverter 2, which is determined by:

$$
i_{2}(t)=\frac{2 n}{\pi}\left(i_{d}(t) \cos \psi+i_{q}(t) \sin \psi\right) .
$$

In Equation (2), the phase shift $\psi$ is the control variable; $i_{d}(t)$ and $i_{q}(t)$ are system states; $v_{2}(t)$ is a state as well as the output; and $v_{1}(t)$ and $i_{R}(t)$ are the disturbances. The Terminal 1 voltage, $v_{1}(t)$, is usually measured in order for the front-end active rectifier to regulate the MVDC bus. In addition, a large capacitor is usually located at the output of the active rectifier to avoid the double line frequency oscillation. For that reason, in one sampling period, the small variation of $v_{1}(t)$ can be assumed to be zero, without loss of the generality.

Next, linearizing Equation (2) around an operating point, a DAB small signal model can be derived as follows:

$$
\frac{d}{d t}\left[\begin{array}{c}
i_{d} \\
i_{q} \\
v_{2}
\end{array}\right]=\left[\begin{array}{ccc}
-\omega_{p} & -\omega_{s} & -\rho_{1} \cos \psi \\
\omega_{s} & -\omega_{p} & -\rho_{1} \sin \psi \\
\rho_{2} \cos \psi & \rho_{2} \sin \psi & 0
\end{array}\right]\left[\begin{array}{c}
i_{d} \\
i_{q} \\
v_{2}
\end{array}\right]+\left[\begin{array}{c}
\rho_{1} V_{2} \sin \psi \\
-\rho_{1} V_{2} \cos \psi \\
\rho_{2} I_{\psi}
\end{array}\right] \varphi-\left[\begin{array}{c}
0 \\
0 \\
1 \\
\overline{C_{o}}
\end{array}\right] i_{R}
$$


where $\rho_{1}=\frac{4 n}{\pi L_{s}} ; \rho_{2}=\frac{2 n}{\pi C_{o}} ;$ and $I_{\psi}=-I_{d} \sin \psi+I_{q} \cos \psi$. Here, the lower case notations of the currents and voltage denote small signal quantities whilst the upper case notations express their values at the equilibrium point:

$$
\left[\begin{array}{c}
I_{d} \\
I_{q}
\end{array}\right]=\frac{4}{\pi L_{s}} \times \frac{1}{\omega_{p}^{2}+\omega_{s}^{2}} \times\left[\begin{array}{cc}
-\omega_{p} & \omega_{s} \\
-\omega_{s} & -\omega_{p}
\end{array}\right] \times\left[\begin{array}{c}
-V_{1}+n V_{2} \cos \psi \\
n V 2 \sin \psi
\end{array}\right] .
$$

In Equation (4), $i_{R}$ is a disturbance. If it can be compensated, the robustness of the voltage controller can be increased, and the bandwidth of the closed-loop system can be expanded. As a consequence, a faster and better dynamic performance can be achieved. Since the target of this paper is to remove the current sensor, $i_{R}$ is thus estimated by using an observer. Therefore, an RPIO is designed in the next section.

\section{Observer Design}

In this section, a Reduced-order Proportional Integral Observer (RPIO) is designed to estimate the load current. A reduced-order topology is preferred due to its simplicity and feasibility in implementation. PIO is chosen due to its ability for disturbance estimation. Compared to nonlinear disturbance observers, RPIO is simpler with fewer design parameters and has a more explicit model that can be used for other purposes, not only feedforward control.

Equation (4) can be expressed as:

$$
\left\{\begin{array}{l}
\dot{x}=A_{11} x+A_{12} v_{2}+B_{1} u+N_{1} d \\
y=A_{21} x+N_{2} d=\dot{v}_{2}-A_{22} v_{2}-B_{2} u
\end{array}\right.
$$

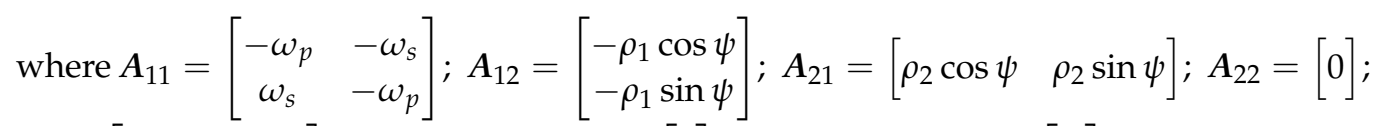
$\boldsymbol{B}_{1}=\left[\begin{array}{c}\rho_{1} V_{2} \sin \psi \\ -\rho_{1} V_{2} \cos \psi\end{array}\right] ; \boldsymbol{B}_{2}=\left[\rho_{2} I_{\psi}\right] ; \boldsymbol{N}_{1}=\left[\begin{array}{l}0 \\ 0\end{array}\right] ; \boldsymbol{N}_{2}=\left[-\frac{1}{C_{0}}\right] ; \boldsymbol{x}=\left[\begin{array}{c}i_{d} \\ i_{q}\end{array}\right] ; \boldsymbol{d}=\left[i_{R}\right] ;$ and $\boldsymbol{u}=[\varphi]$.

The proposed RPIO is then designed as follows:

$$
\left\{\begin{array}{l}
\dot{\hat{x}}=A_{11} \hat{x}+A_{12} v_{2}+B_{1} u+\mathcal{L}_{P}(y-\hat{y})+N_{1} \hat{d} \\
\dot{\hat{d}}=\mathcal{L}_{I}(y-\hat{y}) \\
\hat{y}=A_{21} \hat{x}+N_{2} \hat{d}
\end{array}\right.
$$

where $\hat{x}=\left[\begin{array}{ll}\hat{i}_{d} & \hat{i}_{q}\end{array}\right]^{T} ; \hat{\boldsymbol{d}}=\left[\hat{i}_{R}\right] ; \mathcal{L}_{P}$ and $\mathcal{L}_{I}$ are the observer gain, $\mathcal{L}_{P} \in \mathbb{R}^{2}$ and $\mathcal{L}_{I} \in \mathbb{R}$.

Let $\boldsymbol{e}_{x} \triangleq \boldsymbol{x}-\hat{\boldsymbol{x}}$, and $\boldsymbol{e}_{\boldsymbol{d}} \triangleq \boldsymbol{d}-\hat{\boldsymbol{d}}$. Since the essence of disturbance $\boldsymbol{d}$ is the load current, its dynamic is usually much slower than the sampling frequency due to the large capacitor at the output side. Therefore, its differentiate can be assumed to be zero in one sampling period, i.e., $\dot{d}=0$. Then, the dynamic model of the observation error is:

$$
\left[\begin{array}{c}
\dot{e}_{x} \\
\dot{e}_{d}
\end{array}\right]=\left[\begin{array}{cc}
A_{11}-\mathcal{L}_{P} A_{21} & N_{1}-\mathcal{L}_{P} N_{2} \\
-\mathcal{L}_{I} A_{21} & -\mathcal{L}_{I} N_{2}
\end{array}\right]\left[\begin{array}{c}
e_{x} \\
e_{d}
\end{array}\right]
$$

Denoting $e \triangleq\left[\begin{array}{l}e_{x} \\ e_{d}\end{array}\right] ; \mathcal{A} \triangleq\left[\begin{array}{cc}A_{11} & N_{1} \\ 0 & 0\end{array}\right] ; \mathcal{C} \triangleq\left[\begin{array}{ll}A_{21} & N_{2}\end{array}\right] ;$ and $\mathcal{L} \triangleq\left[\begin{array}{c}\mathcal{L}_{P} \\ \mathcal{L}_{I}\end{array}\right] ;$ then $\mathcal{A} \in \mathbb{R}^{3 \times 3}$, $\mathcal{C} \in \mathbb{R}^{1 \times 3}$ and $\mathcal{L} \in \mathbb{R}^{3 \times 1}$, and Equation (8) is rewritten as:

$$
\dot{e}=(\mathcal{A}-\mathcal{L C}) e
$$


The observability of the error dynamics is verified by checking the rank condition of the observability matrix $\mathcal{O}(\mathcal{A}, \mathcal{C}) \triangleq\left[\begin{array}{lll}\mathcal{C} & \mathcal{C} \mathcal{A} & \mathcal{C} \mathcal{A}^{2}\end{array}\right]^{T}$. Utilizing the system parameters listed in Table A1, it is easy to derive that:

$$
\operatorname{rank}(\mathcal{O}(\mathcal{A}, \mathcal{C}))=3
$$

Since $\mathcal{A} \in \mathbb{R}^{3 \times 3}$, this means that the Equation (9) is observable. Therefore, the PIO gain $\mathcal{L}$ is designed such that matrix $(\mathcal{A}-\mathcal{L C})$ is Hurwitz implying the convergence of the observation error $e$ to zero. This is equivalent to the stability of the matrix $\left(\mathcal{A}^{T}-\mathcal{C}^{T} \mathcal{L}^{T}\right)$ since all eigenvalues of $(\mathcal{A}-\mathcal{L C})$ and $\left(\mathcal{A}^{T}-\mathcal{C}^{T} \mathcal{L}^{T}\right)$ are the same. In addition, the observability of the pair $(\mathcal{A}, \mathcal{C})$ is equivalent to the controllability of the pair $\left(\mathcal{A}^{T}, \mathcal{C}^{T}\right)$. Therefore, numerous methods can be used to design $\mathcal{L}$. In this work, the standard linear-quadratic regulator (LQR) method for designing $\mathcal{L}$ is employed by considering $\mathcal{A}^{T}$ and $\mathcal{C}^{T}$ as a system and input matrix of a dynamical system with the state and input denoted by $v$ and $w$, respectively,

$$
\dot{v}=\mathcal{A}^{T} v+\mathcal{C}^{T} w
$$

and $w=-\mathcal{L}^{T} v$ is the designed feedback controller for Equation (10). Then. consider the following performance index:

$$
J=\int_{0}^{\infty}\left(v^{T} \mathcal{Q} v+w^{T} \mathcal{R} w\right) d t
$$

where $\mathcal{Q} \in \mathbb{R}^{3 \times 3}$ is a positive semidefinite matrix such that $\left(\mathcal{Q}^{1 / 2}, \mathcal{A}^{T}\right)$ is detectable and $\mathcal{R} \in \mathbb{R}, \mathcal{R}>0$. It is well known in the LQR theory that the controller gain, which stabilizes the Equation (10) while minimizing the performance index in Equation (11), is computed by

$$
\mathcal{L}^{T}=\mathcal{R}^{-1} \mathcal{C P},
$$

where $\mathcal{P} \in \mathbb{R}^{3 \times 3}$ is a positive semidefinite matrix, which is the unique solution of the following Riccati equation:

$$
\mathcal{P} \mathcal{A}^{T}+\mathcal{A P}+\mathcal{Q}-\mathcal{P} \mathcal{C}^{T} \mathcal{R}^{-1} \mathcal{C P}=0
$$

Note here that the convergence speed of $v$ is the same as that of $\boldsymbol{e}$ since the eigenvalues of closed-loop systems are the same, as aforementioned. Thus, $\mathcal{Q}$ and $\mathcal{R}$ are designed parameters such that the observation error convergence speed, i.e., the absolute value of the minimum real part of all eigenvalues of $\mathcal{A}^{T}-\mathcal{C}^{T} \mathcal{L}^{T}$, is as desired, usually two to six times bigger than that of the original system [24].

After determining the gain $\mathcal{L}$, the observer can be deployed by applying coordinator transformation (14) to avoid the derivative $\dot{v}_{2}$ in Equation (6):

$$
\left[\begin{array}{l}
\hat{x} \\
\hat{d}
\end{array}\right]=z+\mathcal{L} v_{2},
$$

where $z$ is the new state variable defined by:

$$
\dot{z}=\mathcal{F} \boldsymbol{z}+\mathcal{G} v_{2}+\mathcal{H} \boldsymbol{u} .
$$

and $\mathcal{F}, \mathcal{G}, \mathcal{H}$ are new observer gains and matrices. Block diagram of the proposed RPIO observer with regard to the new state variable is illustrated in Figure 3. Substituting Equation (15) into Equation (6) and Equation (7), $\mathcal{F}, \mathcal{G}$ and $\mathcal{H}$ can be calculated by: 


$$
\left\{\begin{array}{l}
\mathcal{F}=\mathcal{A}-\mathcal{L C} \\
\mathcal{G}=\left[\begin{array}{c}
A_{12} \\
0
\end{array}\right]-\mathcal{L} A_{22}+\mathcal{F} \mathcal{L}, \\
\mathcal{H}=\left[\begin{array}{c}
B_{1} \\
0
\end{array}\right]-\mathcal{L} B_{2} .
\end{array}\right.
$$

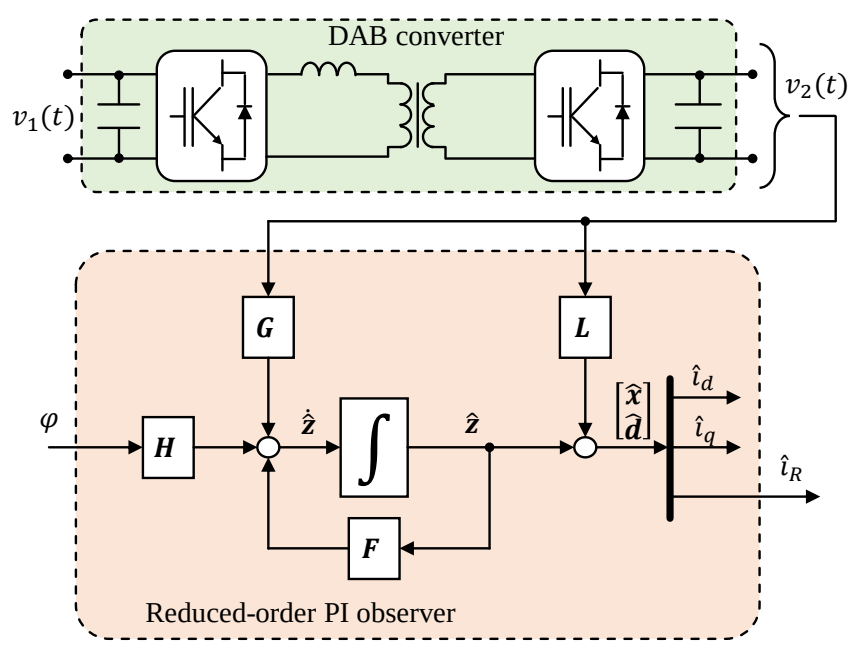

Figure 3. Observer diagram.

\section{Phase Drift Compensation}

In the best case, the observer designed above can estimate the fundamental components of the primary current $i_{p e}(t)$ and the terminal current with the accuracy of $100 \%$. However, in realistic systems, there is a phase drift between the fundamental current and the actual one when the terminal voltages are not matched.

When the terminal voltages are matched, the current waveform is symmetrical. Hence, the zero crossing point of the current and its fundamental component are coincident. However, when $V_{1}$ is other than $n V_{2}$, the current waveform becomes distorted. Accordingly, there will be phase lead $\left(V_{1}<n V_{2}\right)$ or $\operatorname{lag}\left(V_{1}>n V_{2}\right)$ between the fundamental and the actual load angles. Figure 4 demonstrates this effect when $V_{1}=25 \mathrm{~V}$ and $V_{2}=20 \mathrm{~V}$. As seen, in that context, the time lag between the primary current (trapezoidal curve) and the fundamental one (dashed sinusoidal curve) is $2 \mu$ s (i.e., 14.4 degrees in the angular scale). This error is significant and should be compensated.

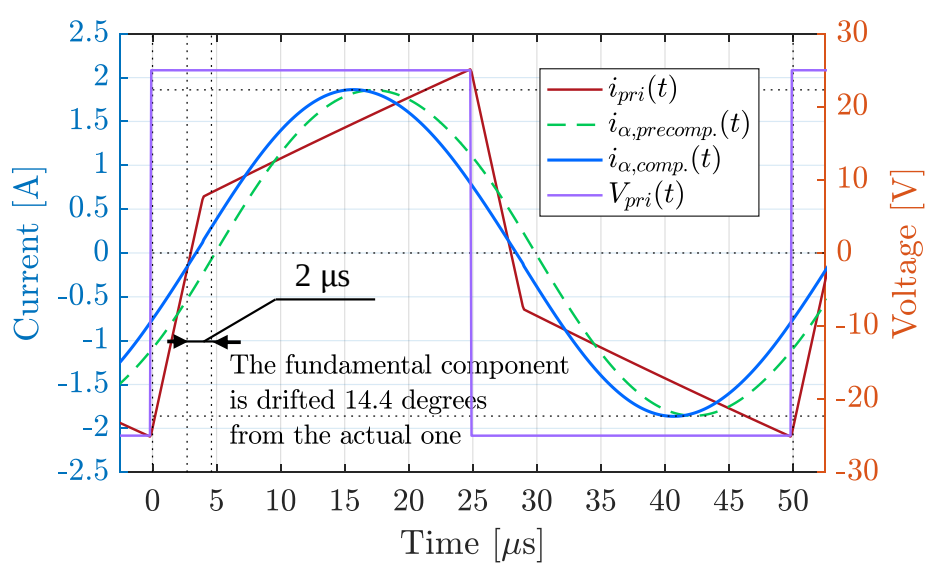

Figure 4. Phase drift effect when terminal voltages are not matched. 
According to [25], the load angle $\phi$ could be estimated by a simple equation:

$$
\phi_{e s t}=\frac{n V_{2}}{V_{1}+n V_{2}}\left(\psi+\frac{V_{1}-n V_{2}}{n V_{2}} \times \frac{\pi}{2}\right) .
$$

As also confirmed in [25], Equation (17) can be used to calculate $\phi$ very accurately with the error less than 2 degrees. Now, denoting $\hat{\phi}$ as the estimated load angle obtained by the proposed observer:

$$
\hat{\phi}=\arctan \frac{\hat{i}_{q}}{\hat{i}_{d}}
$$

Since both $\phi_{\text {est }}$ and $\hat{\phi}$ can be derived easily, there is no difficulty in calculating the load angle erro $\delta$ :

$$
\delta=\hat{\phi}-\phi_{\text {est }}
$$

After that, a vector rotation is applied to correct the error in the observed data. Figure 5 demonstrates the compensation principle. The rotation is accomplished using Equation (20),

$$
\left[\begin{array}{l}
\hat{i}_{d, c o m} \\
\hat{i}_{q, \mathrm{com}}
\end{array}\right]=\mathcal{T}\left[\begin{array}{l}
\hat{i}_{d} \\
\hat{i}_{q}
\end{array}\right],
$$

where $\mathcal{T}$ is the Park transformation matrix, $\mathcal{T}=\left[\begin{array}{cc}\cos \delta & \sin \delta \\ -\sin \delta & \cos \delta\end{array}\right]$. Accordingly, the new system state $z$ should also be updated with regard to the compensation as described in Figure 6 .

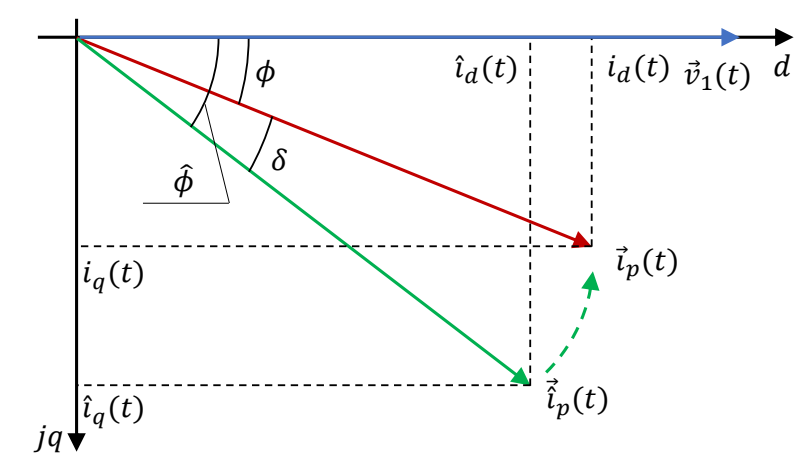

Figure 5. Vector rotation for compensating the phase drift.

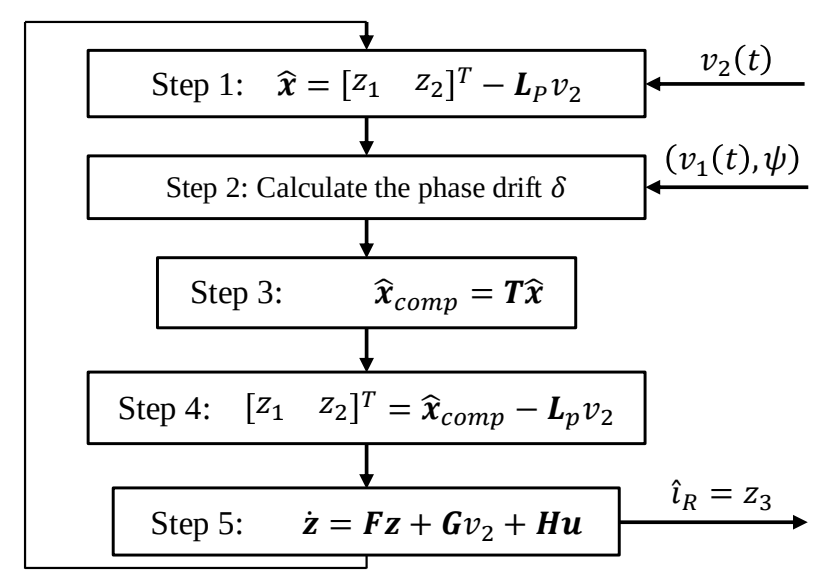

Figure 6. Implementation diagram of the proposed observer with regard to the phase drift compensation. 
Compensation effect is illustrated in Figure 4. By applying Equation (20), the compensated fundamental waveform (continuous sinusoidal curve) is shifted backwards to cancel the phase lag. As a result, the phase error becomes insignificant.

\section{Control System Design}

Figure 7 illustrates the diagram of the whole control system. There are two controllers to be designed: the feedforward current regulator $G_{u}^{-1}(z)$ and the feedback voltage compensator $G_{c}(z)$.

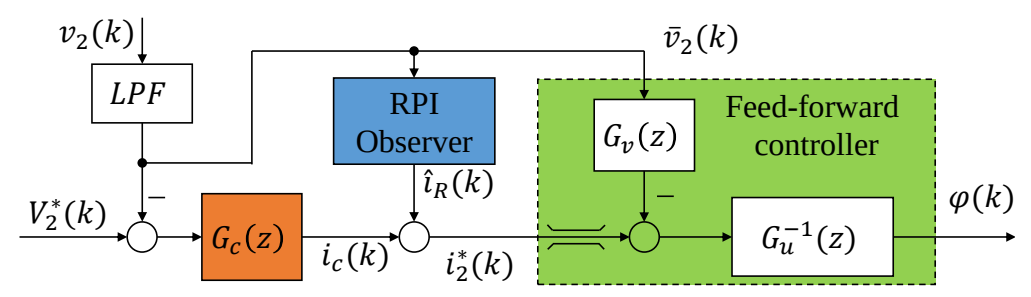

Figure 7. Control system diagram.

\subsection{Feedforward Current Controller}

In the previous section, the load current $i_{R} \equiv d$ is estimated by the RPIO. This section then introduces the feedforward current controller. By linearizing Equation (3) around one operating point, the small signal output current $i_{2}$ can be derived as:

$$
i_{2}=\frac{2 n}{\pi} \cos \psi i_{d}+\frac{2 n}{\pi} \sin \psi i_{q}+\left(-I_{d} \sin \psi+I_{q} \cos \psi\right) \varphi .
$$

Accordingly, the following system model represents the relation from the small signal phase shift $\varphi$ to the small signal output current $i_{2}$ :

$$
\left\{\begin{array}{c}
\dot{z}=\mathcal{F} \boldsymbol{z}+\mathcal{G} v_{2}+\mathcal{H} \boldsymbol{u} \\
i_{2}=\mathcal{M} \boldsymbol{z}+\mathcal{N} v_{2}+\mathcal{D} \boldsymbol{u}
\end{array}\right.
$$

where $\mathcal{M} \triangleq \frac{2 n}{\pi}\left[\begin{array}{lll}\cos \psi & \sin \psi & 0\end{array}\right] ; \mathcal{N} \triangleq \mathcal{M} \mathcal{L}_{P} ; \mathcal{D} \triangleq-I_{d} \sin \psi+I_{q} \cos \psi ;$ and $u=\varphi$.

Equation (22) can be represented in form of transfer functions as Equation (23):

$$
i_{2}(s)=G_{u}(s) u(s)+G_{v}(s) v_{2}(s),
$$

where $G_{u}(s) \triangleq \mathcal{M}(s \mathcal{I}-\mathcal{F})^{-1} \mathcal{H}+\mathcal{D} ; G_{v}(s) \triangleq \mathcal{M}(s \mathcal{I}-\mathcal{F})^{-1} \mathcal{G}+\mathcal{N} ;$ and $\mathcal{I}$ is the identity matrix, $\mathcal{I} \in \mathbb{R}^{3 \times 3}$. From that, the feedforward controller is designed as:

$$
u(s)=\left(i_{2}^{*}(s)-G_{v}(s) v_{2}(s)\right) G_{u}^{-1}(s) .
$$

Since the controller contains the inversion of $G_{u}(s)$, the minimum phase characteristics should be checked.

Figure 8 demonstrates the pole-zero map of $G_{u}(z)$ (discrete version of $G_{u}(s)$ ) with system parameters listed in Table A1. As shown, $G_{u}(z)$ has three poles and three zeros. Among those, there is one zero located outside the unit circle (i.e., non-minimum phase zero) that makes the inverse of $G_{u}(z)$ unstable. Hence, this non-minimum phase zero should be tracked and canceled. This can be done by employing the zero-phase error tracking controller introduced in [26]. 


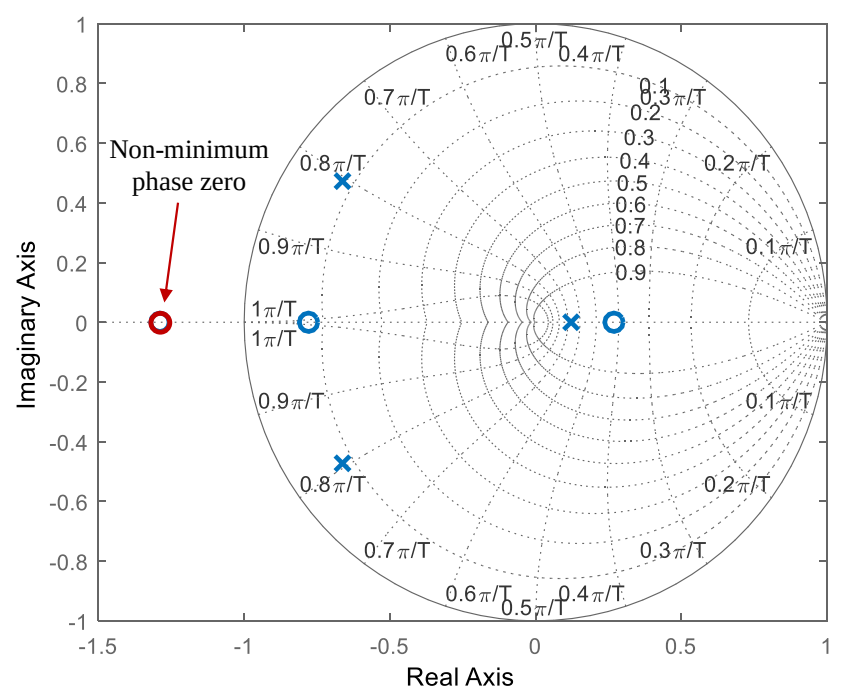

Figure 8. Pole-zero map of $G_{u}(z)$.

First, $G_{u}(z)$ is factorized into two parts: $G_{u}^{a}(z)$, which includes zeros located inside the unit circle that will be poles of the feedforward controller; and $G_{u}^{u}(z)$, which consists of all non-minimum phase zeros:

$$
G_{u}(z)=G_{u}^{a}(z) G_{u}^{u}(z) .
$$

According to reference [26], the non-minimum phase zeros can be tracked and canceled by replacing the non-minimum phase zeros part $G_{u}^{u}(z)$ by $G_{u}^{u *}(z)$, which is defined by:

$$
G_{u}^{u *}(z)=\frac{\left(G_{u}^{u}(1)\right)^{2}}{G_{u}^{u}\left(z^{-1}\right)},
$$

where $G_{u}^{u}(z)=\Sigma_{k=0}^{m} b_{k} z^{k}$ and $G_{u}^{u}\left(z^{-1}\right)=\sum_{k=0}^{m} b_{k} z^{-k}$, and $m$ is the number of non-minimum phase zeros. In this paper, $m=1$.

Consequently, the feedforward controller in established the discrete domain as:

$$
u(k)=\left(i_{2}^{*}(k)-G_{v}(z) v_{2}(k)\right) G_{c f f}(z),
$$

where $G_{c f f}(z)=\left(G_{u}^{a}(z) G_{u}^{u *}(z)\right)^{-1}$.

\subsection{Voltage Controller}

A proportional integral (PI) - typed compensator is intentionally employed for the voltage loop in order to compensate for the steady state error caused by the feedforward current controller.

The closed-loop transfer function of the current loop is represented by:

$$
G_{i i}(z)=\frac{i_{2}(k)}{i_{2}^{*}(k)}=\frac{G_{u}^{u}(z)}{G_{u}^{u *}(z)} .
$$

The transfer function from capacitor current to terminal voltage can be easily established in the $z$-domain using the bilinear transformation:

$$
G_{v i}(z)=\frac{v_{2}(k)}{i_{\mathcal{c}}(k)}=\frac{T_{z}}{2 C_{o}} \times \frac{z+1}{z-1},
$$

where $T_{z}$ is the sampling period. 
From Equations (28) and (29), the voltage loop model can be yielded as Equation (30). Note that one unit delay (represented by $z^{-1}$ ) should be taken into account due to the sampling operation:

$$
G_{v}(z)=z^{-1} G_{i i}(z) G_{v i}(z) G_{L P F}(z),
$$

where $G_{L P F}(z)$ is a low-pass filter. The crossover frequency of the low-pass filter is chosen at one-tenth of the switching frequency to cancel the ripple caused by switching operation.

The PI controller is then designed in the frequency domain by giving a desired phase margin and bandwidth. Here, the crossover frequency is chosen around $50 \mathrm{~Hz}$ intentionally to reduce the double line frequency voltage ripple reflected from the downstream inverter (future application); and the phase margin is chosen around 60 degrees to compensate for steady state error caused by the feedforward controller. Consequently, the PI controller parameters can be calculated.

\section{Simulation and Experimental Results}

The laboratory scaled experiment system is depicted in Figure 9. A summary of system parameters is shown in Table A1. A programmable power supply configured at constant voltage mode is connected to Terminal 1, whereas a DC electronic load is connected to Terminal 2. The winding ratio of the transformer is 1:1. The primary referred leakage inductance and resistance measured at $20 \mathrm{kHz}$ is $67.5 \mu \mathrm{H}$ and $50 \mathrm{~m} \Omega$, respectively.

Aiming to compare among methods, the terminal currents are also measured by using two shunt resistors. The voltages across the resistors are then amplified by isolation amplifiers and filtered out by a low pass filter (LPF). Terminal voltages are also determined in the same manner using voltage divider, isolation amplifier and LPF. The amplifier used in the experiment is a Broadcom ACPL-C79A precision isolation amplifier with $200 \mathrm{kHz}$ bandwidth, hence the amplitude and phase of the measured voltage and current could be ensured.

A TMS320F28335 experiment kit from Texas Instrument is used to deploy the proposed observer and control system designs. Modulation frequency is fixed at $20 \mathrm{kHz}$. Sampling rate is the same as the switching frequency. In order to ensure the accuracy of the measured terminal current (for comparison purpose), the Analog-to-Digital rate is set to $100 \mathrm{kHz}$. The data is then averaged after every five samples.

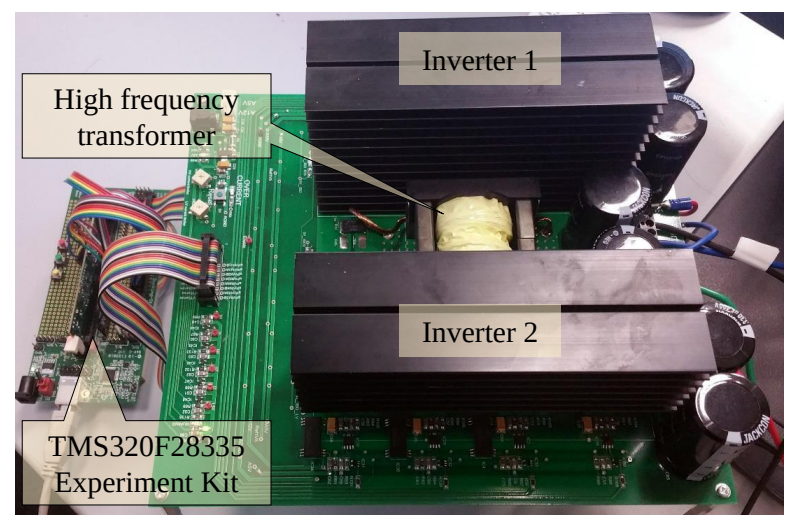

Figure 9. Laboratory-scaled experiment system.

\subsection{Simulation Verification}

Simulation study is conducted to examine the impact of variable changes as well as the influence of designed parameters on the observation performance in the transient state. Figure 10a,b illustrates the simulation results as the phase shift and terminal voltage change, respectively. Input voltage is set at $25 \mathrm{~V}$; the phase shift is 30 degrees. At initialization, the load resistance is nominal at $20 \Omega$, hence output voltage is $25 \mathrm{~V}$ as well. 
Since $\mathcal{R} \in \mathbb{R}$, in this paper, $\mathcal{R}$ is assigned to be 1 to simplify the designing process. Two different matrices $\mathcal{Q}_{1}$ and $\mathcal{Q}_{2}$ are used to find the observer gain and matrices:

$$
\begin{aligned}
& \mathcal{Q}_{1}=5 \times \mathcal{I}, \\
& \mathcal{Q}_{2}=500 \times \mathcal{I},
\end{aligned}
$$

where $\mathcal{I}$ is the $3 \times 3$ identity matrix.

In the first simulation, after $2 \mathrm{~ms}$, the load resistance is suddenly stepped down by $25 \%$ (load increased by 1.33 times) while the phase shift remains constant. As a consequence, output voltage reduces gradually. As seen in Figure 10a, the observer output, either obtained by designing with $\mathcal{Q}_{1}$ (blue curve) or $\mathcal{Q}_{2}$ (violet curve), matches the load current (red curve) within $0.75 \mathrm{~ms}$. When the voltage reduces, the observed current still adheres to the actual one very well.

The phase shift is changed correspondingly to the change of the load in the second simulation. As the load jumps up, the phase shift is also increased to 42 degrees (40\%) to keep the terminal voltage stable. The output of the observer, either obtained by designing with $\mathcal{Q}_{1}$ (blue curve) or $\mathcal{Q}_{2}$ (violet curve), converges after $0.75 \mathrm{~ms}$. There is a steady state error of $0.05 \mathrm{~A}$ (see Figure 10b) in the observed current compared to the simulated one. This means that the observer can estimate the terminal current with the accuracy of approximately $97 \%$.

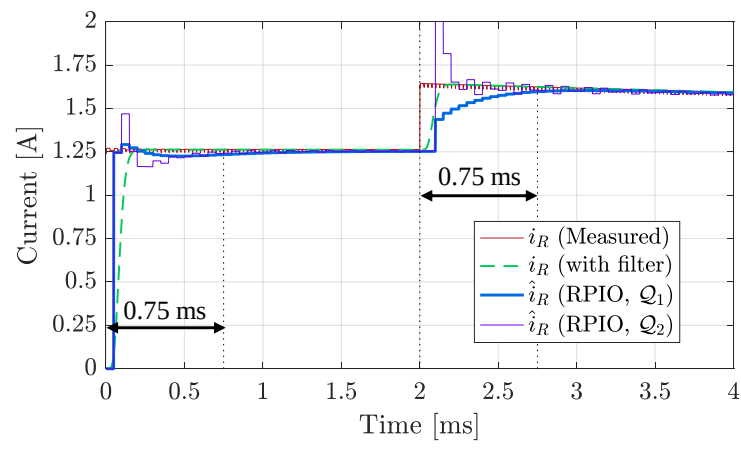

(a)

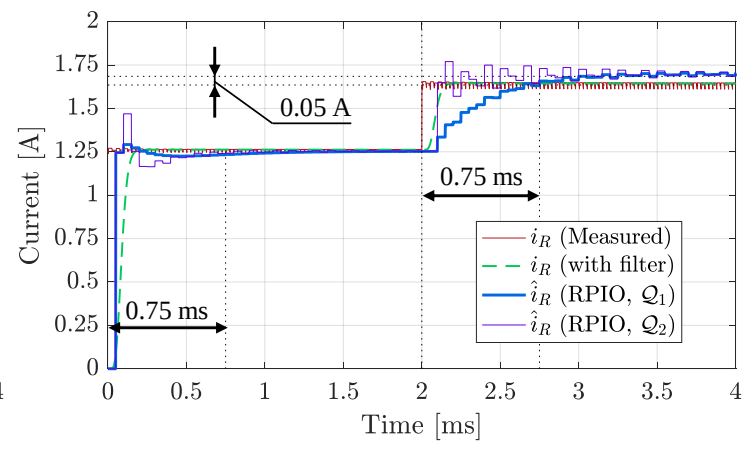

(b)

Figure 10. Simulation responses of the RPIO. (a) $R_{\text {load }}=20 \Omega \Rightarrow 15 \Omega, \psi=30 \mathrm{deg}$; (b) $R_{\text {load }}=20 \Omega \Rightarrow$ $15 \Omega, \psi=30 \mathrm{deg} \Rightarrow 42 \mathrm{deg}$.

In both simulation cases above, the observer designed with $\mathcal{Q}_{2}$ responses much faster than when it is designed with $\mathcal{Q}_{1}$. Theoretical calculation shows that the eigenvalues of the observer obtained by $\mathcal{Q}_{1}$ and $\mathcal{Q}_{2}$ defined above using LQR method are:

$$
E_{1}=\left[\begin{array}{c}
-0.7993+0.5659 j \\
-0.7993-0.5659 j \\
0.8868+0.0000 j
\end{array}\right] \quad \text { and } \quad E_{2}=\left[\begin{array}{c}
-0.7103+0.5036 j \\
-0.7103-0.5036 j \\
0.3216+0.0000 j
\end{array}\right] \text {, }
$$

where $j=\sqrt{-1}$. Since all poles of $E_{2}$ are closer to the origin of the discrete domain than the correspondences of $\boldsymbol{E}_{1}$, it is easy to comprehend the fast response of the RPIO when designed with $\mathcal{Q}_{2}$ rather than with $\mathcal{Q}_{1}$.

The convergence time could be further reduced by increasing the value of matrix $\mathcal{Q}$ in the LQR method, i.e., placing eigenvalues of the observer closer to the origin. However, there is a trade-off between robustness and dynamic response. A higher value of $\mathcal{Q}$ results in a faster but more sensitive observer. On the contrary, a smaller $\mathcal{Q}$ brings more robust observation performance, but slower response time as indicated in Figure 10a,b.

From the above simulation verification, $\mathcal{Q}_{1}$ is chosen as the design parameter for better robustness of the proposed RPIO. Accordingly, observer gains are: 


$$
\mathcal{L}_{P}=\left[\begin{array}{c}
-0.3082 \\
1.3735
\end{array}\right] \quad \text { and } \quad \mathcal{L}_{I}=[-2.0891] .
$$

Substituting the above $\mathcal{L}_{P}$ and $\mathcal{L}_{I}$ into Equation (16), then matrices $\mathcal{F}, \mathcal{G}, \mathcal{H}$ can be yielded as listed in Table A2. Note that, although Equation (16) is derived in the continuous domain, it can also be used for deriving $\mathcal{F}, \mathcal{G}$, and $\mathcal{H}$ without losing the correctness.

\subsection{Open-Loop Experimental Verification}

This experiment is conducted to verify the static performance of the proposed observer. Figure 11a,b illustrates the output responses of the proposed observer (RPIO), designed with $\mathcal{Q}=\mathcal{Q}_{1}$ defined above, in comparison with the actual values and with the reduced order observer (ROBS), which was designed in [20] based on the Terminal 1 current under some different operating conditions.

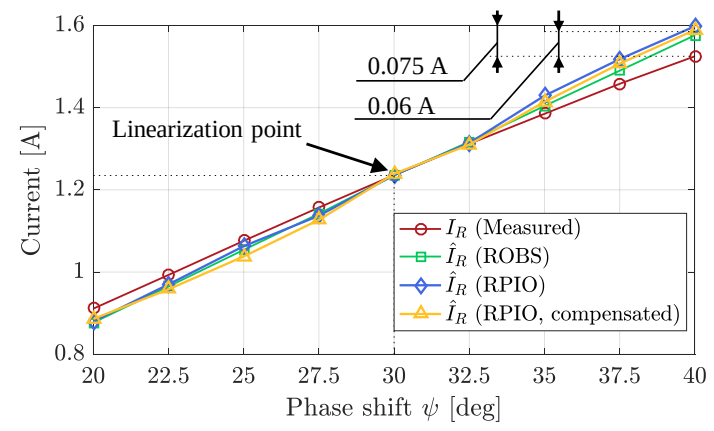

(a)

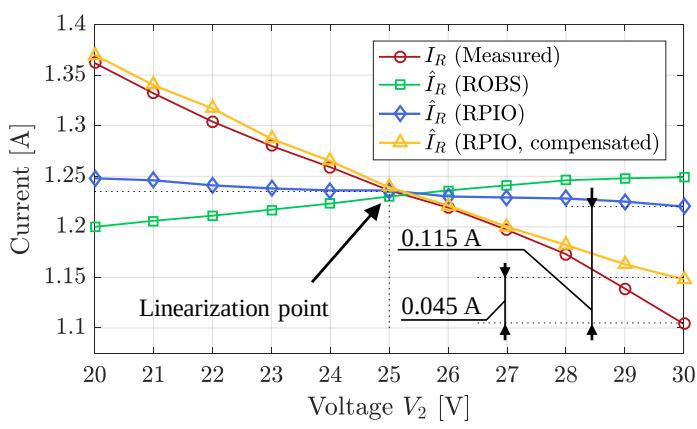

(b)

Figure 11. Static performance of the proposed observer. (a) $\psi=20 \sim 40 \mathrm{deg} ; V_{2}=25 \mathrm{~V}$; (b) $V_{2}=20 \sim 30 \mathrm{~V}$; $\psi=30 \mathrm{deg}$.

First, Terminal 2 voltage is fixed at its nominal by setting the DC electronic load to constant voltage mode. The phase shift is then gradually increased from 20 to 40 degrees $( \pm 33 \%$ around the linearization point). As shown in Figure 11a, all four curves are very close to each other. The estimation error is unremarkable when $\psi \leq 30$ degrees. The error is greatest when $\psi$ is 40 degrees. At that condition, the measured current (red, circle marked) is about $1.53 \mathrm{~A}$, whereas the estimates by ROBS (green, square marked) and RPIO (blue, diamond marked) are $1.58 \mathrm{~A}$ and $1.60 \mathrm{~A}$, respectively. In other words, the estimation error is about $5 \%$. Because the error is already small, applying the compensation in Equation (20) does not contribute to a big but minor reduction on the estimation error to $4 \%$. This result is similar to the simulation study described above.

Next, Terminal 2 voltage is varied with a step of $1 \mathrm{~V}$ while fixing other parameters. Results shown in Figure 11b suggest that, in practice, the observation performance seems to be more sensitive to the voltage changes than the phase shift changes. The error is about $\pm 0.15 \mathrm{~A}$ when applying ROBS. Employing RPIO (non-compensated) helps reduce the error to $\pm 0.115 \mathrm{~A}$, which is still quite remarkable. This is because of the phase drift phenomenon mentioned in the previous section. By using the proposed compensation method, the estimation error is much more reduced. The maximum error is now only $0.045 \mathrm{~A}$, which is more than three times reduction compared to that obtained by ROBS.

Another quantitative comparison can be conducted regarding the $R^{2}$ coefficient (coefficient of determination). Accordingly, when varying the phase shift while keeping terminal voltages constant (Figure 11a), the $R^{2}$ of the three observations are $99.94 \%$ (RBOS), $99.70 \%$ (RPIO) and $99.62 \%$ (RPIO with phase drift compensated). There is no significant differences between the three observer results. Nevertheless, when terminal voltages are not matched, RPIO brings a remarkable performance improvement. With the experimental data shown in Figure $11 \mathrm{~b}$, the $R^{2}$ are $95.74 \%$ (RBOS), $97.66 \%$ (RPIO) and $98.22 \%$ (RPIO with phase drift compensated). Obviously, 
applying the proposed RPIO can help to improve the observation accuracy by approximately $2 \%$. If the phase drift phenomenon is compensated, the improvement is even higher at nearly $2.5 \%$. That is to say, the proposed observer accompanied with the compensation method can estimate the Terminal 2 current with the accuracy of more than $98 \%$.

Although containing some division and trigonometric functions, compensator in Equation (20) executes pretty fast on DSP F28335. At a $150 \mathrm{MHz}$ system clock, the observer (including compensation procedures) takes only $2.86 \mu$ s to accomplish. The executing time can be further reduced by optimizing the programming or using a lookup table for trigonometric functions. Since the sampling time is $50 \mu \mathrm{s}$, there is plenty of time for other functions and algorithms such as PI calculation or communication, etc. This also provides possibility to increase the sampling frequency to more than $100 \mathrm{kHz}$ if required.

\subsection{Feedforward Current Loop}

With $\mathcal{Q}=\mathcal{Q}_{1}$ and other observer gains and matrices as listed in Table A2, the feedforward current controller is derived as shown in Table A3. In this experiment, the DC electric load is still configured to the constant voltage mode at $25 \mathrm{~V}$. Only the current loop is examined. At first, the reference current is set to the nominal of $1.235 \mathrm{~A}$. After some time, it is increased by $20 \%$ to 1.5 amperes. The current responses are then displayed in Figure 12.

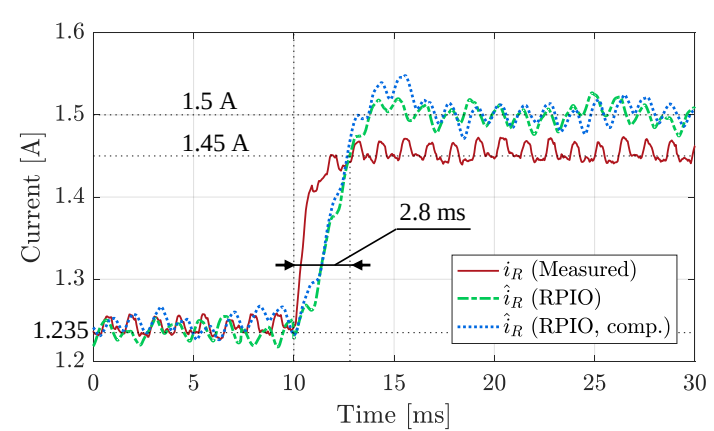

(a)

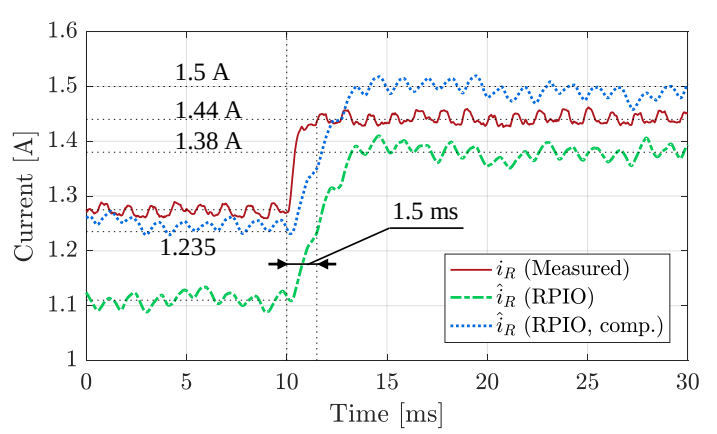

(b)

Figure 12. Experimental current response. (a) current responses when $V_{2}=25 \mathrm{~V}$; (b) current responses when $V_{2}=20 \mathrm{~V}$.

The measured current (denoted by the red, solid curve) is well stabilized around $1.235 \mathrm{~A}$ (i.e., at the nominal condition). In this context, the observer can also estimate the current very precisely. The dashed (original RPIO) and dotted (RPIO with compensation) curves are mostly coincident with the actual one in the interval from 0 to $10 \mathrm{~ms}$ (Figure 12a). When the reference current steps up, the current responds quickly to reach its stable state after $2.8 \mathrm{~ms}$. Although the observation values match the set-point, the actual average current is only $1.45 \mathrm{~A}$. Therefore, a steady state error of $0.05 \mathrm{~A}$ exists. Since the voltages across two terminals are equal, there is no significant difference between the original RPIO and RPIO with compensation performances.

Figure $12 \mathrm{~b}$ shows the current response when terminal voltages are not matched (e.g., $V_{1}=25 \mathrm{~V}$ and $V_{2}=20 \mathrm{~V}$ ). When the current command is $1.235 \mathrm{~A}$, the terminal current is about $1.275 \mathrm{~A}$. When the command increases to $1.5 \mathrm{~A}$, the terminal current settles down after $1.5 \mathrm{~ms}$ at $1.44 \mathrm{~A}$. The steady state errors are $+0.04 \mathrm{~A}$ and $-0.06 \mathrm{~A}$, respectively, which are relatively small $(\leq 4 \%)$. As for the observed current, the observation error is remarkable without compensation as discussed above. When the compensation method is applied, the same result as the one obtained when the voltage ratio is unity can be achieved.

\subsection{Voltage Loop}

In this section, the dynamic performance of the voltage control system is investigated. There are two experiments: load increase and load decrease. The Terminal 1 voltage is fixed at $25 \mathrm{~V}$. The DC 
electronic load operates in the constant resistance mode. The reference for the voltage control loop is $25 \mathrm{~V}$. Both static and dynamic performances of the voltage loop are considered in two cases: with and without the feedforward path. The output of the RPIO is employed for feeding forward to the output of the voltage controller that makes the system a feedforward controller (FFC). Note here that the estimated load current $\hat{i}_{R}$ is phase-drift-compensated. In the second case, the feedforward path is ignored, and hence the control system behaves as a normal voltage mode controller (VMC). In both cases, the same PI controller as shown in Table A3 is employed.

At first, the resistance is set to $25 \Omega$, which is 1.25 times greater than its nominal $(20 \Omega)$. After some time, the resistance is suddenly reduced to $13.3 \Omega(-33.5 \%$ reduction). Figure 13 a shows the voltage and current waveforms in the full-time scale and Figure 13b provides a zoomed-in view of the transient period. As seen, the voltage is well regulated regardless of with or without the feedforward path. When the load suddenly increases, there is a sag of $1.05 \mathrm{~V}$ (i.e., $4 \%$ of the set-point) in the voltage responses when VMC is employed. After $11 \mathrm{~ms}$, the voltage is restored and stabilized at the desired value. Since the voltage controller is of the PI type, there is no steady state error. When a feedforward path from the RPIO is applied, dynamic performance is significantly improved. The voltage fluctuation is mostly suppressed as the maximum variation is only $\pm 0.35 \mathrm{~V}$, or $1.4 \%$ in the per unit (pu.) scale. The settling time is also much reduced from $11 \mathrm{~ms}$ to only $4.5 \mathrm{~ms}$.

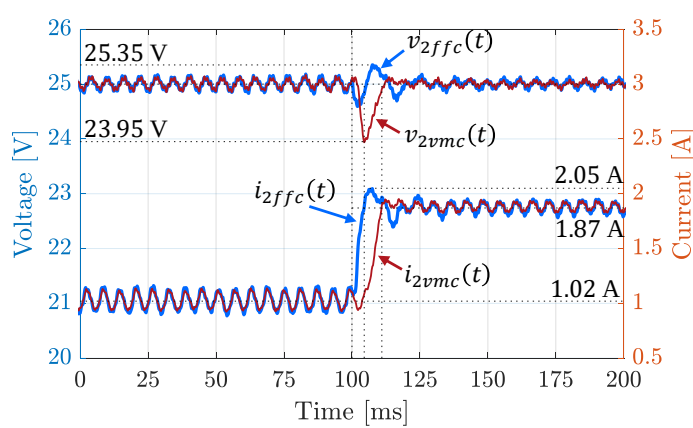

(a)

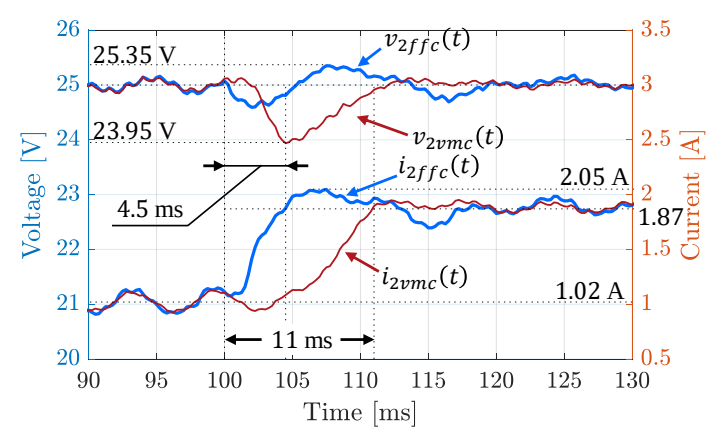

(b)

Figure 13. Experimental voltage and current responses: load increases. (a) full-time scale; (b) zoomed-in view of the transient period.

The same effect is observed when decreasing the load. In this experiment, the load change scenarios are reversed. The load resistance is initialized at $13.3 \Omega$ then after a while is suddenly increased to $25 \Omega$. Figure $14 \mathrm{a}, \mathrm{b}$ shows the voltage and current waveforms in the full-time and zoomed-in scales.

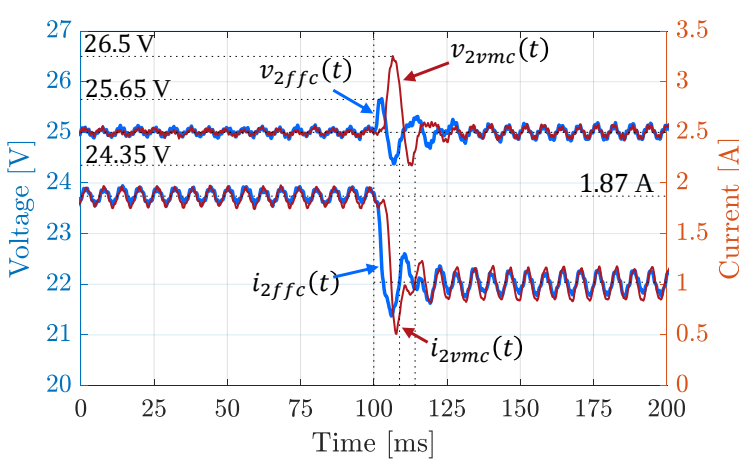

(a)

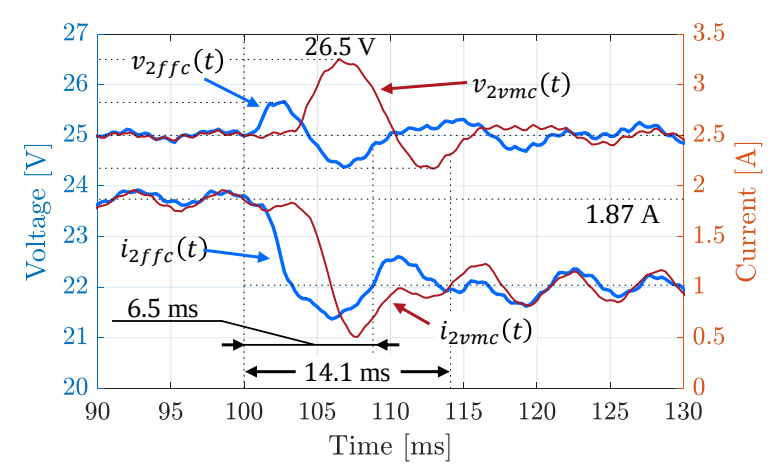

(b)

Figure 14. Experimental voltage and current responses: load decreases. (a) full-time scale; (b) zoomed-in view of the transient period. 
Without the feedforward path, the voltage swell is $1.5 \mathrm{~V}$ or $6 \%$ in the per unit scale. The settling time is also relatively long at $14.1 \mathrm{~ms}$. By utilizing our proposed control system, the voltage variation reduces more than $50 \%$ to only $\pm 0.65 \mathrm{~V}$ (or $2.6 \%$ in pu.). Response speed is about two times faster as the voltage settles down after only $6.5 \mathrm{~ms}$.

\section{Conclusions}

This paper proposes a new observer-based control system for the dual-active-bridge converter. More specifically, a reduced-order proportional integral observer (RPIO) is designed to estimate the terminal current instead of measuring it. In addition, a compensation technique is introduced to reduce the phase drift phenomenon due to the current distortion waveform. Simulation and experimental results show that the observation accuracy is more than $98 \%$. Compared to the observer designed in $[20,21]$, the observation performance is significantly improved.

A current feedforward and voltage feedback control system are then developed to regulate the Terminal 2 voltage. As a consequence, the dynamic performance is remarkably improved. The voltage fluctuation can be almost ignored and the response speed is two times faster.

Moreover, the proposed RPIO can estimate not only terminal current, but also the load angle, the direct and the quadrature components of the AC current, with high accuracy. Those estimated values can also be used for other control topologies-for example, the decoupled current control [20]. Therefore, not only is the system dynamic enhanced, but the overall efficiency can also be improved by reducing the reactive power (proportional to the quadrature current).

The above outcomes are achieved with only the information of the terminal voltages. Besides the economical benefit of removing current sensors, efforts for increasing the processing speed and suppressing the noisy current signal are also eliminated. Furthermore, the implementation of the proposed RPIO is also simple and feasible as the computing time (including phase drift compensation) is only $2.86 \mu \mathrm{s}$. This means that this technique can be applied for a much faster dual-active-bridge converter system.

Author Contributions: Duy-Dinh Nguyen proposed the idea, and performed experiments; Dinh-Hoa Nguyen designed the observer; Goro Fujita and Toshihisa Funabashi helped complete the rough idea; all members contributed significantly in writing and revising the paper.

Conflicts of Interest: The authors declare no conflict of interest.

\section{Appendix A. Parameters}

Table A1. System parameters.

\begin{tabular}{cccc}
\hline Parameter & Symbol & Value & Unit \\
\hline Trans. ratio & $n$ & $1: 1$ & \\
Inductance & $L_{S}$ & 67.5 & $\mu \mathrm{H}$ \\
Resistance & $R_{s}$ & 50 & $\mathrm{~m} \Omega$ \\
Nom. frequency & $F_{S}$ & 20 & $\mathrm{kHz}$ \\
Nom. phase shift & $\Psi$ & 30 & $\mathrm{deg}$ \\
Sampling time & $T_{z}$ & 50 & $\mu \mathrm{s}$ \\
Dead-time & $T_{d}$ & 1 & $\mu \mathrm{s}$ \\
DC capacitors & $C_{1}, C_{2}$ & 1000 & $\mu \mathrm{F}$ \\
\hline
\end{tabular}


Table A2. Observer parameters.

\begin{tabular}{|c|c|c|c|}
\hline Parameter & \multicolumn{3}{|c|}{ Value } \\
\hline $\mathcal{Q}$ & & {$\left[\begin{array}{lll}5 & 0 & 0 \\
0 & 5 & 0 \\
0 & 0 & 5\end{array}\right]$} & \\
\hline $\mathcal{L}_{P}$ & {$[-0.3$} & $3082 \quad 1.37$ & $735]^{T}$ \\
\hline $\mathcal{L}_{I}$ & & -2.0891 & \\
\hline $\mathcal{F}$ & {$\left[\begin{array}{c}-0.8036 \\
0.5591 \\
0.0162\end{array}\right.$} & $\begin{array}{l}-0.5731 \\
-0.7959 \\
-0.0142\end{array}$ & $\left.\begin{array}{c}-0.0180 \\
0.0813 \\
0.8878\end{array}\right]$ \\
\hline $\mathcal{G}$ & {$[-0.1386$} & -3.0853 & $0.2067]^{T}$ \\
\hline $\mathcal{H}$ & {$[7.0032$} & 1.2379 & $0.2045]^{T}$ \\
\hline
\end{tabular}

Table A3. Controller parameters.

\begin{tabular}{cc}
\hline Parameter & Value \\
\hline$G_{c f f}(z)$ & $\frac{0.13513(z+0.7758)\left(z^{2}+1.608 z+0.9706\right)}{z^{2}(z+0.7791)}$ \\
\hline$G_{c}(z)$ & $\frac{0.25125(z-0.99)}{z-1}$ \\
\hline$G_{L P F}(z)$ & $\frac{0.3859 z}{z-0.6141}$ \\
\hline
\end{tabular}

\section{References}

1. Ari, G.; Baghzouz, Y. Impact of high PV penetration on voltage regulation in electrical distribution systems. In Proceedings of the 2011 International Conference on Clean Electrical Power (ICCEP), Ischia, Italy, 14-16 June 2011; pp. 744-748.

2. Divan, D.; Johal, H. Distributed FACTS-A new concept for realizing grid power flow control. IEEE Trans. Power Electron. 2007, 22, 2253-2260. [CrossRef]

3. She, X.; Huang, A.Q.; Burgos, R. Review of solid-state transformer technologies and their application in power distribution systems. IEEE J. Emerg. Sel. Top. Power Electron. 2013, 1, 186-198. [CrossRef]

4. Zhao, B.; Song, Q.; Liu, W.; Sun, Y. Overview of dual-active-bridge isolated bidirectional DC-DC converter for high-frequency-link power-conversion system. IEEE Trans. Power. Electron. 2014, 29, 4091-4106. [CrossRef]

5. Qin, H.; Kimball, J.W. Closed-loop control of DC-DC dual-active-bridge converters driving single-phase inverters. IEEE Trans. Power Electron. 2014, 29, 1006-1017.

6. Segaran, D.; Holmes, D.G.; McGrath, B.P. Enhanced load step response for a bidirectional DC-DC converter. IEEE Trans. Power Electron. 2013, 28, 371-379. [CrossRef]

7. Zhao, B.; Song, Q.; Liu, W.; Sun, W. Current-stress-optimized switching strategy of isolated bidirectional DC-DC converter with dual-phase-shift control. IEEE Trans. Ind. Electron. 2013, 60, 4458-4467. [CrossRef]

8. Zhao, B.; Song, Q.; Li, J.; Liu, W.; Liu, G.; Zhao, Y. High-frequency-link dc transformer based on switched capacitor for medium-voltage DC power distribution application. IEEE Trans. Power Electron. 2016, 31, 4766-4777. [CrossRef]

9. Dutta, S.; Hazra, S.; Bhattacharya, S. A Digital Predictive Current-Mode Controller for a Single-Phase High-Frequency Transformer-Isolated Dual-Active Bridge DC-to-DC Converter. IEEE Trans. Ind. Electron. 2016, 63, 5943-5952. [CrossRef]

10. Song, W.; Hou, N.; Wu, M. Virtual Direct Power Control Scheme of Dual Active Bridge DC-DC Converters for Fast Dynamic Response. IEEE Trans. Power Electron. 2018, 33, 1750-1759. [CrossRef] 
11. Engel, S.P.; Soltau, N.; Stagge, H.; De Doncker, R.W. Dynamic and balanced control of three-phase high-power dual-active bridge DC-DC converters in DC-grid applications. IEEE Trans. Power Electron. 2013, 28, 1880-1889. [CrossRef]

12. Engel, S.P.; Soltau, N.; Stagge, H.; De Doncker, R.W. Improved instantaneous current control for high-power three-phase dual-active bridge DC-DC converters. IEEE Trans. Power Electron. 2014, 29, 4067-4077. [CrossRef]

13. Pahlevaninezhad, M.; Eren, S.; Pahlevani, H.; Askarian, I.; Bagawade, S. Digital Current Sensorless Control of Current-Driven Full-Bridge DC/DC Converters. IEEE Trans. Power Electron. 2018, 33, 1797-1815, [CrossRef]

14. Zhang, Q.; Min, R.; Tong, Q.; Zou, X.; Liu, Z.; Shen, A. Sensorless predictive current controlled DC-DC converter with a self-correction differential current observer. IEEE Trans. Ind. Electron. 2014, 61, 6747-6757. [CrossRef]

15. Cimini, G.; Ippoliti, G.; Orlando, G.; Pirro, M. Current sensorless solution for PFC boost converter operating both in DCM and CCM. In Proceedings of the 2013 21st Mediterranean Conference on Control \& Automation (MED), Plata-nias-Chania, Crete, 25-28 June 2013; pp. 137-142.

16. Xiong, F.; Wu, J.; Liu, Z.; Hao, L. Current Sensorless Control for Dual Active Bridge DC-DC Converter with Estimated Load-Current Feedforward. IEEE Trans. Power Electron. 2018, 33, 3552-3566. [CrossRef]

17. She, X.; Huang, A.Q.; Ni, X. Current sensorless power balance strategy for DC/DC converters in a cascaded multilevel converter based solid state transformer. IEEE Trans. Power Electron. 2014, 29, 17-22. [CrossRef]

18. She, X.; Yu, X.; Wang, F.; Huang, A.Q. Design and demonstration of a 3.6-kV-120-V/10-kVA solid-state transformer for smart grid application. IEEE Trans. Power Electron. 2014, 29, 3982-3996. [CrossRef]

19. Ge, J.; Zhao, Z.; Yuan, L.; Lu, T. Energy feed-forward and direct feed-forward control for solid-state transformer. IEEE Trans. Power Electron. 2015, 30, 4042-4047. [CrossRef]

20. Nguyen, D.D.; Fujita, G.; Bui-Dang, Q.; Ta, M.C. Reduced-order Observer-based Control System for Dual-Active-Bridge DC/DC Converter. IEEE Trans. Ind. Appl. 2018, [CrossRef]

21. Nguyen, D.D.; Fujita, G. Observer-based decoupling power control for frequency modulated Dual-Active-Bridge converter. In Proceedings of the 2016 IEEE 8th International Power Electronics and Motion Control Conference (IPEMC-ECCE Asia), Hefei, China, 22-26 May 2016; pp. 754-760.

22. Qin, H.; Kimball, J.W. Generalized average modeling of dual active bridge DC-DC converter. IEEE Trans. Power Electron. 2012, 27, 2078-2084.

23. Nguyen, D.D.; Fujita, G. Sensorless control of Dual-Active-Bridge DC/DC converter based on reduced-order observer. In Proceedings of the 2018 IEEE International Power Electronics Conference and ECCE Asia (IPEC \& ECCE), Niigata, Japan, 20-24 May 2018.

24. Lewis, F.L.; Vrabie, D.; Syrmos, V.L. Optimal Control; John Wiley \& Sons: Hoboken, NJ, USA, 2012.

25. Nguyen, D.D.; Nguyen, D.T.; Fujita, G. New Modulation Strategy Combining Phase Shift and Frequency Variation for Dual-Active-Bridge Converter. IEEJ J. Ind. Appl. 2017, 6, 140-150. [CrossRef]

26. Tomizuka, M. Zero phase error tracking algorithm for digital control. J. Dyn. Syst. Meas. Control 1987, 109, 65-68. [CrossRef]

(C) 2018 by the authors. Licensee MDPI, Basel, Switzerland. This article is an open access article distributed under the terms and conditions of the Creative Commons Attribution (CC BY) license (http://creativecommons.org/licenses/by/4.0/). 